\title{
Watching dogs chase their tails to understand OCD
}

Repetitive behaviors, often seen in human neuropsychiatric disorders such as autism and obsessive compulsive disorder (OCD), have not been studied extensively, even though such compulsive behaviors occur in multiple species including dogs. Several similarities have been found between compulsive behavior in dogs and humans, suggesting that they may share a neurobiological basis.

Many dog breeds exhibit spontaneous compulsive behaviors, often diagnosed as canine compulsive disorder, which can include repetitive pacing, tail-chasing, chasing shadows or lights, freezing and staring. Researchers led by Hannes Lohi of University of Helsinki in Finland looked for possible associations between tail-chasing and environmental factors and personality. The team conducted a questionnaire study of the owners of nearly 400 bull terriers, miniature bull terriers, German shepherds and Staffordshire bull terriers, breeds in which compulsive tail-chasing is most common (PLoS One 7, e41684; 2012).

Tail chasers were more likely to suffer from other compulsive behaviors, including freezing or trance-like behavior, than the

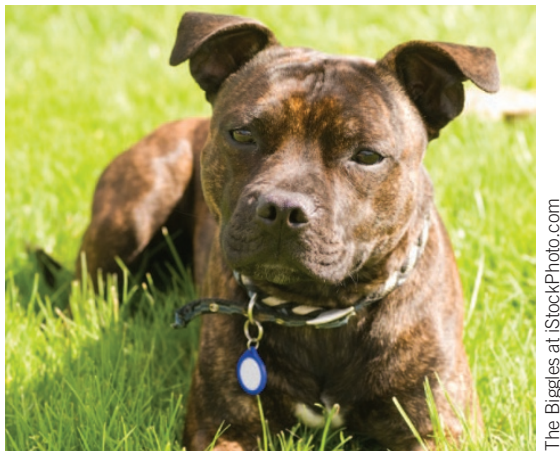

non-tail chasers. The team hypothesized that the trance-like "staring episodes" seen in these dogs could be analogous to similar behaviors in humans with autism spectrum disorders. The frequency of tailchasing behavior showed a large variation in the dog populations, similar to OCD, in which symptoms and co-occurrence of compulsive behaviors vary greatly.

Early separation from the mother and poor maternal care of the puppy were found to predispose dogs to tail-chasing. Childhood trauma or stress has been associated with OCD, suggesting that both dog and human compulsive behaviors have similar environmental causes. The scientists also found an association between tail-chasing and personality, particularly in the male dogs they studied. Tail chasers were generally shyer and less aggressive towards humans than non-tail chasers. Shyness in dogs shares characteristics with behavioral inhibition, a human temperament style involving restraint, withdrawal and avoidance of new things, that is characteristic of many OCD patients.

Tail-chasing behavior was found to be connected with the dogs' nutrient intake: dogs that received dietary vitamin and mineral supplements had less tail-chasing compared to the dogs not receiving any nutrients. "Interestingly, similar preliminary results have been observed in human OCD," said Katriina Tiira, first author of the study, in a press release. Vitamin B6 in particular was found to be beneficial to the dogs.

The study suggests that dogs with canine compulsive disorder offer an excellent animal model for studying the genetic background and environmental factors associated with the human compulsive behaviors seen in autism and OCD.

Kara Rosania

\section{EASIER ESTIMATION OF AN INDIVIDUAL'S 'BODY TIME'}

For many organisms, including humans, the activity of certain biological processes and the expression of certain genes and proteins fluctuate predictably over a period of roughly 24 hours. These oscillations are maintained by an internal time-keeping mechanism called the circadian clock. 'Body time' refers to the setting of each individual's circadian clock, which can vary by as much as 12 hours among individuals. Body time can also be influenced by working late-night shifts, suffering from jet lag or lacking regular routines (e.g., sleep, meal times). These individual variations are important because body time affects not only personal comfort and stress levels, but also potency and toxicity of some medications as well as the likelihood of developing diet-induced obesity. Hence, reliable determination of an individual's body time is necessary to facilitate chronotherapy (or matching of drug delivery to body time) and time-restricted diet strategies.

The conventional method for body time determination involves repeated blood sampling for the measurement of cortisol or melatonin over 24 hours or more under controlled environmental conditions. This approach has the advantage of measuring body time directly, but it is time- and labor-intensive and somewhat invasive. As an alternative, a group of Japanese scientists led by Takeya Kasukawa (RIKEN Center for Developmental Biology, Hyogo) and Masahiro Sugimoto (Keio University, Yamagata, and Kyoto University) developed a molecular timetable method for estimating body time. The circadian oscillation patterns of specific genes or metabolites in the body are profiled in detail to create a reference timetable of metabolites whose abundance fluctuates over 24 hours. Scientists can then measure these metabolites in only two samples, taken 12 hours apart, from an individual and compare the measurements to the reference timetable to estimate that individual's body time. Kasukawa and Sugimoto's team previously applied their molecular timetable method to estimating body time in mice using expression of clock-controlled genes and blood plasma metabolites. They now report application of their technique to estimating individual body time in humans.

On the basis of the two anti-phase blood samples, the scientists were able to accurately pinpoint individuals' body times to within 3 hours, even under different experimental conditions (Proc. Natl. Acad. Sci. USA published online 27 August 2012; doi:10.1073/ pnas.1207768109). “Our study demonstrates that human internal body time can be detected using limited time-point sampling and a reference metabolite timetable," wrote the authors. They hope that their technique "may lead to highly optimized and personalized medicine in the future." 